\title{
Harlequin Baby: the Challenge of Pain Management in the Neonatal Period
}

\author{
Mark David S. Yu ${ }^{1}$ and Grace G. Catalan ${ }^{2}$ \\ ${ }^{1}$ Department of Anesthesiology, Philippine General Hospital, University of the Philippines Manila \\ ${ }^{2}$ Department of Anesthesiology, College of Medicine and Philippine General Hospital, University of the Philippines Manila
}

\begin{abstract}
This case is about a 35-week neonate diagnosed with harlequin ichthyosis. The neonate was managed by a multidisciplinary team composed of specialists from neonatology, genetics, dermatology, plastic surgery, ophthalmology, rehabilitation medicine, and anesthesiology.
\end{abstract}

Harlequin ichthyosis is characterized by defective keratinization and desquamation of the epidermis which is very painful. Pain management in the neonate entails a proper balance between pain relief and avoidance of serious adverse effects which is a major challenge for caregivers.

Key Words: harlequin ichthyosis, neonatal pain

\section{Introduction}

Harlequin ichthyosis is a genetic disorder characterized by defective keratinization and desquamation of the epidermis.

The incidence is 1:1,000,000 births and death is usually due to dehydration, sepsis, respiratory failure, hypothermia, hypoglycemia, or renal failure. Key elements of treatment include humidified incubator, thermoregulation, nutrition, skin and eye care, pain control, physiotherapy, and infection control. Little has been reported about the holistic management of these patients in the neonatal period. ${ }^{1}$

Evidence suggests that infants, particularly preterm infants, are more sensitive to pain. During this vulnerable period of neuronal plasticity, noxious stimuli, in the form of strong painful procedures or repeated mildly painful procedures may trigger long-term changes affecting the structure and function of their nervous system, altered pain response and may contribute to the development of chronic pain. ${ }^{2}$

A major challenge in optimal pain management of neonates is competent pain assessment. ${ }^{3}$ The pain assessment tool should be multidimensional, measuring both physiologic and behavioral parameters. Complex management of these patients can be best achieved by using a multidisciplinary approach characterized by strong communication, both among the medical team and with the family.

Corresponding author: Mark David S. Yu, MD

Department of Anesthesiology

Philippine General Hospital

University of the Philippines Manila

Taft Ave. Ermita, Manila, 1000 Philippines

Telephone: +6320917 7063898

Email: markdavidyu@gmail.com
This paper reports a case of harlequin ichthyosis on the multi-faceted management of the disease in the inpatient setting. It elucidates the role of anesthesia in pain relief, as well as special considerations in pain management in the neonate period. This experience may be of use to assist future physicians in taking care of these patients.

The prevention of pain in neonates is expected. However, there are major gaps in our knowledge regarding the most effective way to accomplish this. Although it may not be possible to completely eliminate pain in neonates, much can be done to reduce the intensity of pain. Given the rarity of the disease and its management challenges, this report offers to assist future teams taking care of these special patients.

\section{Case description}

The index case is a 35 -week preterm male infant who was born to a 31-year-old G3P3 (3003) by normal spontaneous delivery assisted by a midwife at a local birthing center. Birth weight was 2,700 grams and the antenatal period was uneventful. The mother did not have regular prenatal check-ups and no vitamin supplements were taken during pregnancy. The patient was born to a couple with no family history of skin disorder or any similar condition. He was received at the Pediatric emergency room with good activity, good cry, and the following vital signs: heart rate of 120 and respiratory rate of 40 . The patient had minimal retractions and an oxygen saturation of $92 \%$ at room air. Intravenous access was secured. Diagnostics taken included complete blood count, lipid profile, electrolytes, BUN, creatinine, blood culture and sensitivity, and arterial blood gas.

On physical examination (Figure 1), there were generalized thick hyperkeratotic plates over his entire body and scalp as well as deep erythematous fissures. His face was distorted because of taut scales and his ears were bound to the scalp by tight keratinous skin obscuring the pinnae. He also had severe ectropion and eclabium. His hands and feet were tight and edematous with contracted digits showing dusky tapering at distal ends and decreased capillary refill. Extremities showed decreased range of motion, especially in the digits, wrists, elbows, knees, and ankles secondary to skin tautness.

The patient was managed by the departments of neonatology, genetics, dermatology, plastic surgery, ophthalmology, and anesthesiology. Key elements of the 
treatment plan during the neonatal period included skin care, sepsis monitoring and treatment, pain control, and management of ectropion and digital contractures.

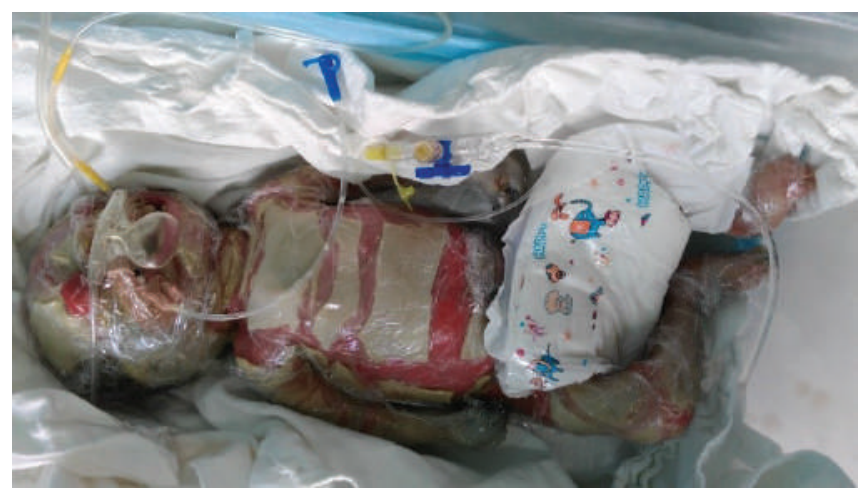

Figure 1. Patient's actual photo.

The patient was stable from birth on room air, but was maintained on oxygen face mask at 6 liters per minute. Feeding consisted of expressed breast milk via orogastric tube. He was maintained with D7.5 IMB computed appropriately to cater to the increased metabolic demand.

Dermatologic care consisted of acitretin per orogastric tube, application of white petrolatum over the entire body surface every 6 hours and urea 10\% lotion. Ophthalmology recommended artificial tears to be applied to both eyes every hour and Lacryvisc gel applied around the eyes three times per day to manage ectropion and prevent secondary keratitis. Slit lamp examination and fundoscopy showed no significant findings. Patient was wrapped using saran wrap to prevent insensible fluid loss and dehydration. Warming lights were placed to assist in thermoregulation. Plastic surgery recommended conservative management of digital contractures with thin sterile gauze rolls soaked in saline solution placed in the palms and soles to prevent contracture progression. Rehabilitation medicine suggested soft splinting of the hands and feet. Genetics was consulted and discussed nature and chance of recurrence in future offspring with the parents.

Pain service initiated a regimen to reduce physiologic stress on the neonate: fentanyl $1 \mathrm{mcg} \quad(\sim 0.4 \mathrm{mcg} / \mathrm{kg})$ intravenously every 4 hours and paracetamol 20mg (7.5 $\mathrm{mg} / \mathrm{kg}$ ) intravenously every 8 hours.

Given the patient's increased susceptibility to infection from his skin condition and known history of preterm delivery, he was closely monitored for sepsis throughout his hospital stay. Despite these efforts, work up revealed positive blood cultures and the patient succumbed to sepsis on the $7^{\text {th }}$ day of life.

\section{Discussion}

It is the basic right of every individual, irrespective of age or size, to have alleviation of pain. Pain in newborn infants is a ubiquitous phenomenon.

The neurobiology of neonatal pain processing, especially in preterm infants, differs significantly from older infants, children, adolescents, and adults. During normal development, pain transmission and modulation undergo rapid growth beginning at 22 weeks gestation; achieving mature functioning at approximately 2 months of age. In contrast to adults, a preterm infant's afferent pain pathway has a higher density of high threshold $A \delta$ and low threshold $\mathrm{A} \beta$ mechanoreceptors that respond with lower firing frequency. On the other hand, neurotransmitters in the inhibitory arm, found within the descending pathway, are immature and will not develop until 4 to 8 weeks later in postnatal life. This translates to greater pain sensitivity and intensity in preterm infants because afferent excitatory pain neurotransmitters, which are plentiful at birth, are not balanced by descending inhibitory neurotransmitters. ${ }^{5}$ Prolonged or repeated pain may increase the response elicited by future painful stimuli (hyperalgesia) and even by usually non-painful stimuli (allodynia). Temporal summation and hyperalgesia can last for as long as 30 to 60 minutes.

Table 1. Commonly used pain assessment tools in neonates

\begin{tabular}{|c|c|c|c|}
\hline Measure & Variables Included & Type of Pain & Psychometric Testing \\
\hline $\begin{array}{l}\text { PIPP (Premature Infant } \\
\text { Pain Profile) }{ }^{22}\end{array}$ & $\begin{array}{l}\text { Heart rate, oxygen saturation, facial actions, } \\
\text { takes state and gestational age into account }\end{array}$ & Procedural, postoperative (minor) & $\begin{array}{l}\text { Reliability, validity, clinical } \\
\text { utility well established }\end{array}$ \\
\hline $\begin{array}{l}\text { NIPS (Neonatal Infant } \\
\text { Pain Score) })^{23}\end{array}$ & $\begin{array}{l}\text { Facial expression, crying, breathing patterns, } \\
\text { arm and leg movements, arousal }\end{array}$ & Procedural & Reliability, validity \\
\hline $\begin{array}{l}\text { NFCS (Neonatal Facial } \\
\text { Coding System) })^{23}\end{array}$ & Facial actions & Procedural & $\begin{array}{l}\text { Reliability, validity, clinical } \\
\text { utility, high degree of } \\
\text { sensitivity to analgesia }\end{array}$ \\
\hline $\begin{array}{l}\text { N-PASS (Neonatal Pain, } \\
\text { Agitation, and } \\
\text { Sedation Scale) })^{24}\end{array}$ & $\begin{array}{l}\text { Crying, irritability, behavioural state, facial } \\
\text { expression, extremity tone, vital signs }\end{array}$ & Postoperative, procedural, ventilated & $\begin{array}{l}\text { Reliability, validity, includes } \\
\text { sedation end of scale, does not } \\
\text { distinguish pain from agitation }\end{array}$ \\
\hline $\begin{array}{l}\text { CRIES (Cry, Requires oxygen, } \\
\text { Increased vital signs, } \\
\text { Expression, Sleeplessness) }\end{array}$ & $\begin{array}{l}\text { Crying, facial expression, sleeplessness, } \\
\text { requires oxygen to stay at }>95 \% \text { saturation, } \\
\text { increased vital signs }\end{array}$ & Postoperative & Reliability, validity \\
\hline COMFORT Scale ${ }^{26}$ & $\begin{array}{l}\text { Movement, calmness, facial tension, } \\
\text { alertness, respiration rate, muscle tone, } \\
\text { heart rate, blood pressure }\end{array}$ & $\begin{array}{l}\text { Postoperative, critical care, developed for } \\
\text { sedation, recently validated for postoperative } \\
\text { pain in } 0 \text { - to 3-year-old infants }\end{array}$ & $\begin{array}{l}\text { Reliability, validity, clinical } \\
\text { utility }\end{array}$ \\
\hline
\end{tabular}


Harlequin Baby

Table 2. Neonatal pain management guidelines

\begin{tabular}{|c|c|c|c|c|c|c|}
\hline Procedure & $\begin{array}{c}\text { Supportive } \\
\text { (where applicable) }\end{array}$ & Oral sucrose & Morphine & Paracetamol & $\begin{array}{c}\text { Local } \\
\text { Anesthesia }\end{array}$ & Remarks \\
\hline NGT/OGT insertion & Sw & $\checkmark$ & & & & \\
\hline Venepuncture/IV cannulation & Sw, NNS, BF & $\checkmark$ & & & & \\
\hline IM/SC Injections/vaccinations & Sw, NNS, BF, SSC & $\checkmark$ & & & & \\
\hline Heel prick & Sw, NNS, BF, SSC & $\checkmark$ & & & & \\
\hline Plaster removal & Sw, NNS, BF, SSC & & & & & Use adhesive remover \\
\hline Dressing change & Sw, NNS, BF, SSC & $\checkmark$ & & & & \\
\hline UVC/UAC & & & & & & Do not clamp or stitch skin \\
\hline Arterial puncture/line & Sw, NNS, BF & $\checkmark$ & & & & \\
\hline PCVC & Sw, NNS & $\checkmark$ & & & & \\
\hline Lumbar puncture & & $\checkmark$ & & & EMLA & Use max $1 \mathrm{~g}$ per procedure \\
\hline ET suction & & & $\checkmark$ bolus & & & \\
\hline Elective ET intubation & & & $\checkmark$ bolus & & & $0.1 \mathrm{mg} / \mathrm{kg}$ only in presence of doctor \\
\hline ROP screen/eye exam & Sw, NNS, BF & $\checkmark$ & & & & \\
\hline ICD insertion & & & $\checkmark$ bolus & & & \\
\hline Post-op laser/caput pain & & & & $\checkmark$ Q4-6H & & \\
\hline Post hernia repair & & & & $\checkmark$ Q4-6H & & \\
\hline Post-op Major surgery & & & $\checkmark$ infusion & & & At least 72 hours post-op \\
\hline Suprapubic tap & NNS & $\checkmark$ & & & & \\
\hline Mechanical ventilation & & & & & & $\begin{array}{l}\text { Consider phenobarb. } \\
\text { Morphine on case by case basis }\end{array}$ \\
\hline Bladder catheterization & NNS & $\checkmark$ & & & & \\
\hline Suture removal & NNS & $\checkmark$ & & & & \\
\hline
\end{tabular}

Sw: swaddling; NNS: non-nutritive sucking; SSC: skin to skin contact; BF: breast feeding

Oral sucrose: Maximum 8 times in 24 hours and three times per procedure. Do not use on babies who are sedated or have poor suck. Do not use for pacifying or settling baby. Local anesthetic: EMLA cream to be used cautiously ing G6PD deficiency.

Reference: Adapted from Lago P, Garetti E, Merazzi D, Pieragostini L, Ancora G, Pirelli A, et al. Guidelines for procedural pain in the newborn. Acta Paediatr. 2009;98:932-939.

During this vulnerable period of neuronal plasticity, noxious stimuli, in the form of strong painful procedures or repeated mildly painful procedures may trigger long-term changes. ${ }^{6}$ Undermanaged pain in preterm infants alters the structure and function of their nervous system which can contribute to the development of chronic pain. Damaged tissue was found to have prominent dendritic sprouting in local sensory nerve terminals causing lowered threshold to pain and hyperinnervation.

A major challenge in optimal pain management of neonates is competent pain assessment. The pain assessment tool should be multidimensional, measuring both physiologic (heart rate, respiratory rate, blood pressure, oxygen saturation, vagal tone, palmar sweating, and plasma cortisol or catecholamine concentrations) and behavioral (changes in facial expressions, body movements, and crying) indicators of pain, because neonates cannot verbalize pain. Prolonged pain causes striking changes in these parameters. During episodes of prolonged pain, neonates enter a "state of passivity" with few, if any, body movements; an expressionless face; decreased heart rate and respiratory variability; and decreased oxygen consumption, all suggestive of a marked conservation of energy. ${ }^{7}$

Selecting the most appropriate tool for evaluating neonatal pain is essential to its management. Documentation of pain is also crucial as there can be variation in pain perception in babies between various caregivers. Many pain scoring tools exist and a few that are used commonly are given in Figure 2. ${ }^{8}$

Pain in newborn infants is a ubiquitous phenomenon. All newborns, even normal ones, will experience iatrogenic pain in the first days of life, commencing with vitamin $\mathrm{K}$ injection and blood collection for sugar and bilirubin or metabolic screening before discharge from the hospital. Neonates admitted to present day neonatal intensive care units (NICU) are constantly exposed to pain, discomfort, or noxious stimuli of variable intensity for a variety of reasons. These include major surgical procedures, needle pricks for blood drawing and cannulations. All these events, especially in preterm infants individually or cumulatively, result in adverse sequelae in the form of death, poor neurologic outcomes, and abnormal somatization and response to pain later in life. ${ }^{8}$

The deep cutaneous fissures found in harlequin ichthyosis are particularly painful, making pain management an important issue in the care of these patients. Unfortunately, the neonates who are at greatest risk of neurodevelopmental impairment because of preterm birth are also those most likely to be exposed to the greatest number of painful stimuli, creating a double-hit effect. ${ }^{9}$

In the management of moderate to severe pain, systemic analgesia using opioid is the mainstay. Opioids have been 
(A)

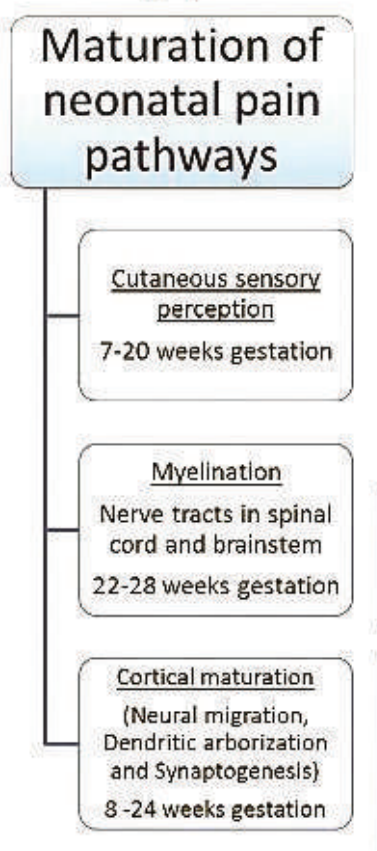

(B)

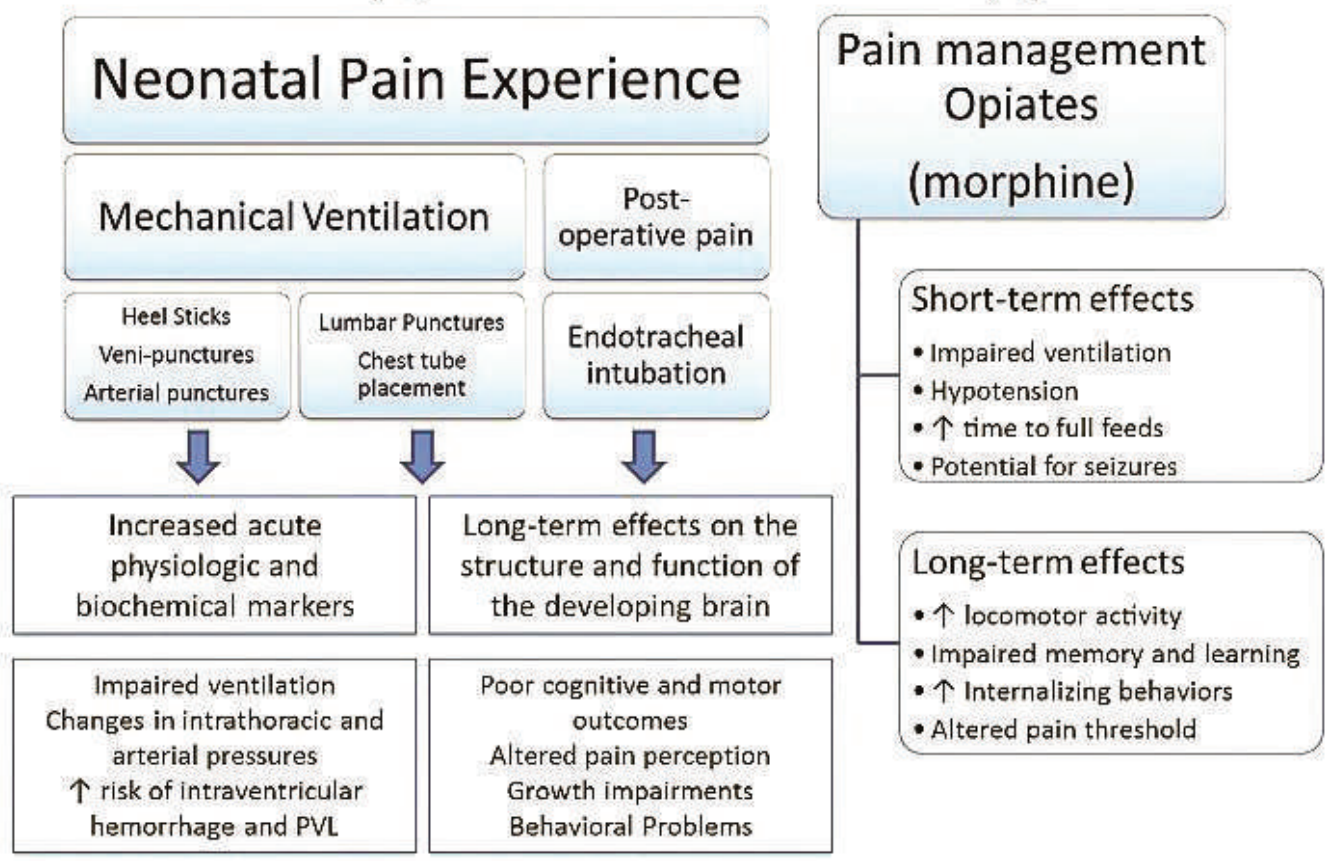

Figure 2. Schematic representation depicting the developmental maturation of pain pathway in neonates.

found beneficial for the clinical stability of critically ill preterm neonates. The half-life of fentanyl in neonates is prolonged, ranging from 317 minutes to 1,266 minutes compared to adults whose range is 222 minutes. Studies have shown that respiratory depression occurs when fentanyl doses are more than $5 \mathrm{mcg} / \mathrm{kg}$. Fentanyl provides rapid analgesia, maintains hemodynamic stability, blocks endocrine stress responses, and prevents pain-induced increases in pulmonary vascular resistance. ${ }^{10}$ Although much about analgesics in neonates is already known, further research is required to ensure that the recommended doses for pain relief in neonates are evidence-based..$^{11}$

Striking a proper balance between effective pain relief and avoidance of serious adverse effects from pain medications is a major challenge for caregivers particularly the anesthesiologist.

Another aspect to consider is parent-child interaction in the early neonatal period, particularly skin-to-skin contact which has been repeatedly shown to be essential for building the foundation of the parent-child relationship. Daily management continues to remain complex even after discharge. Thus, it is important that a social worker be involved early on to help structure a comprehensive discharge plan that will provide the caretakers with support during the transition from hospital to home. ${ }^{12}$

\section{Conclusion}

The role of the multidisciplinary team involves not only catering to the physical health of the patient but also includes issues affecting the parental bonding and home life.
Harlequin icthyosis is a severe disorder of the skin that presents a formidable challenge for even the most skilled perinatal teams. Nevertheless, complex management of these patients can be best achieved by using a multidisciplinary approach characterized by strong communication, both among the medical team and with the family.

\section{Statement of Authorship}

All authors have approved the final version submitted.

\section{Author Disclosure}

All authors declared no conflict of interest.

\section{Funding Source}

This paper was partially funded by the Philippine General Hospital and the authors.

\section{References}

1. Harvey HB, Shaw MG, Morrell DS. Perinatal management of harlequin ichthyosis: a case report and literature review. J Perinatol. 2010; 30(1):66-72.

2. Schwaller F, Fitzgerald M. The consequences of pain in early life: injury-induced plasticity in developing pain pathways. Eur J Neurosci. 2014; 39(3):344-52.

3. Witt N, Coynor S, Edwards C, Bradshaw H. A guide to pain assessment and management in the neonate. Curr Emerg Hosp Med Rep. 2016; 4:1-10.

4. Jamison RN, Edwards RR. Integrating pain management in clinical practice. J Clin Psychol Med Settings. 2012; 19(1):49-64. 
5. Hatfield LA. Neonatal pain: What's age got to do with it? Surg Neurol Int. 2014; 5(Suppl 13):S479-89.

6. Schwaller F, Fitzgerald M. The consequences of pain in early life: injuryinduced plasticity in developing pain pathways. Eur J Neurosci. 2014; 39(3):344-52.

7. Barrington KJ, Batton D, Finley G, Wallman C. Prevention and management of pain in the neonate: an update. Paediatr Child Health. 2007; 12(2):137-8

8. Krishnan L. Pain relief in neonates. J Neonatal Surg. 2013; 2(2):19.

9. Prasad AN. Harlequin baby. Med J Armed Forces India. 2011;67(1):88-9.
10. Pacifici GM. Clinical pharmacology of fentanyl in preterm infants: a review. Pediatr Neonatol. 2015; 56(3):143-8.

11. Harrison D, Yamada J, Stevens B. Strategies for the prevention and management of neonatal and infant pain. Curr Pain Headache Rep. 2010;14(2):113-23.

12. Mosayebi Z, Javidpour M, Rahmati M, et al. The effect of kangaroo mother care on pain from heel lance in preterm newborns admitted to neonatal intensive care unit: a crossover randomized clinical trial. J Compr Ped. 2014; 5(4):e22214

\section{The}

National Elealtur
Science Jourmal

[s nom thdexed tin

SciVerse Scoprus。

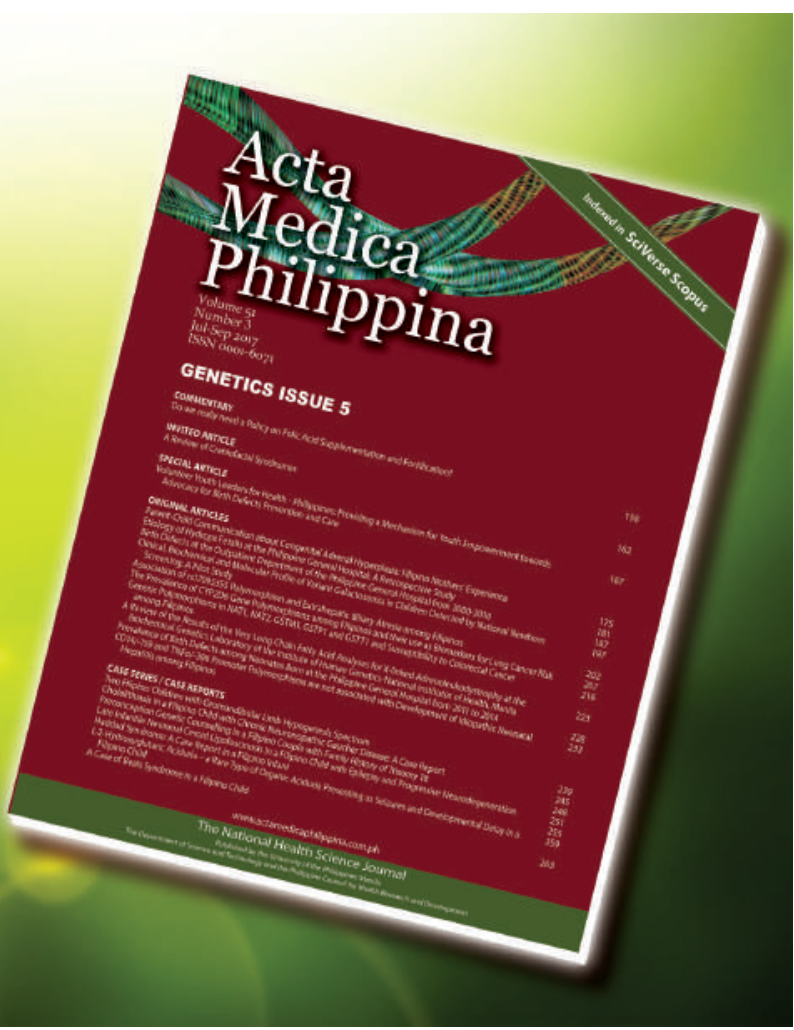

